\title{
Container route optimization in a sea-rail intermodal network
}

\author{
Y. ZHAO \& X. ZHANG \& Q.W. XUE \\ School of Traffic and Transportation, Beijing Jiaotong University, Beijing, China
}

\begin{abstract}
KEYWORD: Sea-rail intermodal; Container route; Genetic algorithm
ABSTRACT: This paper focuses on the problem of computing container routes in an intermodal transportation network which is considered as a multimodal multicommodity network flow problem (MMNFP). According to the characteristics of sea-rail intermodal networks in China, a container routing model is proposed taking into consideration the transportation costs and transfer costs as a 0-1 programming model. The genetic algorithm is implemented, coded, and computationally tested on realistic size networks with promising results. Simulation results show that the algorithm generates optimal routes for each cargo from railway stations to seaports, which provide comprehensive guidance for scientific decisions.
\end{abstract}

\section{INTRODUCTION}

With the development of global economy, the freight demand has grown steadily over the past half century. As a result, intermodal movements are playing a more and more important role in international freight transportation system. Thus, it is essential to compute the optimal routes in intermodal network to minimize the freight cost as much as possible. So far, some researchers have done relative research on the problem.

Min dealt with multiple objectives and on-time service requirements, he developed a chanceconstrained goal programming model which minimizes cost and risk, and at the same time satisfies various on-time service requirements (Min 1991). This study focused on the problem: how to select best routes for shipments through the international intermodal network. The international intermodal routing problem was formulated as a multiobjective multimodal multicommodity flow problem (MMMFP) with time windows and concave costs (Chang 2008). The paper focused on the routing of the tens of thousands of intermodal freight movements and the GIS technology was used to select appropriate intermodal routes from origins and destinations in intermodal networks (Worth \& Peterson 2000). Some scholars assumed the travel time was assumed deterministic and dependent on the departure time, searching optimal routes in networks (Miller \& Mahmassani 2000, Pattanamekar \& Park 2003). The above label correcting algorithm was developed for determining least time paths from all nodes of an intermodal network to a single destination node for every transport mode and departure times assuming time-dependent arc travel times and switching delays between modes (Ziliaskopoulos \& Wardell 2000). A decomposition scheme was proposed for solving the travel planning problem i.e., optimum path problem in a transportation network with deterministic timedependent travel times under the constraint of visiting a sequence of specified nodes (Berube \& Potvin 2006). A backward label setting algorithm was proposed for identifying the entire set of nondominated solutions for the all to one multiple criteria time-dependent shortest path problem with positive cost values for waiting at the nodes (Hamacher \& Ruzika 2006). Zhang considered the transportation costs and transportation time, and the best routes selection problem was transformed into a bi-objective shortest path problem through constructing the virtual network (Zhang \& Wang 2013). Based on multi-commodity flow model, Ji built the car flow assignment and routing optimization model of railway network, which subjected to each car flow moving on one route only, car flow with big volume, the capacity of lines and stations (Ji \& Lin 2011). But there are few papers that combine route selection and transfer selection in a sea-rail intermodal network. This paper based on previous papers build the container routes model considering route selection and transfer selection simultaneously. 
This paper is organized as follows. Section 2 describes the proposed model. Section 3 uses a smallscale network to demonstrate the proposed model. Section 4 concluded the usability of the work presented in this paper.

\section{MODEL FORMULATION}

Let $G=(N, A)$ denote a directed transportation network where $N$ is the set of nodes and $A$ is a set of arcs. Let $O$ be the set of original stations, $O \subseteq N, D$ be the set of destination ports, $D \subseteq N$ and $H$ is the set of intermodal transfers, where the cargo switches its transportation modes, $H \subseteq N$. Let $S$ denotes the set of vessels from transfers to destination ports. For each arc $(i, j)$, the unit cost, $c_{i j}$, is assumed to be a non-negative linear function of the travelling distance between node $i$ and node $j$ transported by trains, $i, j \in N / D$. For each $\operatorname{arc}(h, d)$ The unit cost, $c_{h d}^{s}$, is also assumed to be a nonnegative linear function of the travelling distance from transfer $h$ to port $d$ by vessel $s$. For each transfer node $h, c_{h}^{1}$ is the unit cost that one TEU is transferred from trains to vessels at node $h$.

\section{Notations}

Index and sets

$N$ the set of nodes

$A$ the set of arcs

$O$ the set of original stations for each cargo

$H$ the set of intermodal transfers, where the cargo is transferred from trains to vessels.

$D$ the set of destination ports for each cargo

$i, j$ two nodes, $i, j \in N / D$

$o$ an original station, $\quad o \in O$

$h$ an intermodal transfer, $h \in H$

$d$ a destination port, $d \in D$

$S$ the set of vessels $s \in S$

Parameters

$q_{o d}$ the transportation volume of the cargo od, TEUs

$c_{i j}$ the unit cost of transportation between $i$ and $j$

$c^{s}{ }^{s}$ the unit cost of transportation by vessel $s$ between $h$ and $d$

$c_{h}^{l}$ the unit cost of transferring at $\mathrm{h}$

Decision variables

$x_{\text {od }}^{i j}$ denotes whether the $\operatorname{arc}(i, j)$ is on the optimal route of the cargo od.

$x^{h s}{ }_{\text {od }}$ denotes whether the cargo od is transported by vessel $s$ from $h$ to port $d, s \in S$.

\section{Mathematical formulation}

Problem statement and assumption

The container route problem is the combination of two subproblems: rail container route and sea container route. Thus, the two decision variables $x_{o d}^{i j}, x^{h s}$ od represent the rail container route and sea container route respectively. For each commodity od, $x_{o d}^{i j}$ denotes whether the arc $(i, j)$ is on the optimal rail route. And $x^{h s}$ od denotes whether the vessel $s$ from $h$ to $d$ is selected for the cargo od. Every pair od is treated as a distinct commodity, which is associated with an origin station $o$ and a destination port $d$. Assume that the transportation volume $q_{o d}$ should be sent from $o$ to $d$. So the network $G=(N, A)$ defined above is a multimodal multicommodity network. And the problem is considered as a multimodal multicommodity network flow problem (MMNFP) modeled as a 0-1 programming model.

The problem is formulated based on the following assumptions.

Assumption 1: a cargo can only be transferred at intermodal transfer node.

Assumption 2: the empty container repositioning is not considered explicitly.

Assumption 3: the capacity of arcs among stations and arcs between transfer nodes and destination ports is assumed to be infinite.

Objective 
The objective of the model is to minimize the following objective function $J$ :

$$
\begin{aligned}
J= & \sum_{o \in O, d \in D} q_{o d}\left(\sum_{i, j \in N / D} c_{i j} x_{o d}^{i j}+\sum_{h \in H} \sum_{s \in S} c_{h d}^{s} x_{o d}^{h s}\right) \\
& +q_{o d} \sum_{h \in H} c_{h}^{1} \sum_{s \in S} x_{o d}^{h s}
\end{aligned}
$$

Hence, the first item of the objective represents the transportation cost, which sums up every arc transportation cost of each cargo; the second item denotes transfer cost.

The objective function $J$ is subject to constraints: for each cargo od, it is subject to flow conservation constraint at node $i, i \in N / D$; each destination $d$ must be subject to flow conversation constraint; decision variables constraint.

Constraints

$$
\begin{aligned}
& \sum_{j} x_{o d}^{i j}-\sum_{j} x_{o d}^{j i} \\
& =\left\{\begin{array}{lr}
1, & i=o \\
0, & i \neq o \text { and } i \neq h \\
-1, & i=h
\end{array}\right.
\end{aligned}
$$

$\forall i \in N / D, o \in O, d \in D$

$\sum_{o} \sum_{s} q_{o d} x_{o d}^{h s}=\sum_{o} \sum_{i} q_{o d} x_{o d}^{i h}, \quad \forall h \in H, d \in D$

$\sum_{h} \sum_{s} x_{o d}^{h s}=1, \quad \forall o \in O, d \in D$

$\sum_{i} x_{o d}^{i h}=\sum_{s \in S} x_{o d}^{h s}, \quad \forall o \in O, d \in D, h \in H$

$x_{o d}^{i j}=0$ or $1, \quad \forall i, j \in N / D, o \in O, d \in D$

$x_{o d}^{h s}=0$ or $1, \quad \forall o \in O, d \in D, h \in H, s \in S$

Here, constraint (2) denotes each cargo od at node $\mathrm{i}$ is subject to flow conservation, $i \in N / D$, which ensures every cargo only chooses one route to transport. For cargo od, there is only one flow transported to other nodes at node $o$, so the value of the equation is 1; For intermediate nodes, the number of flow sent to node $i$ equals the number of flow sent from node $i$, so the value of the equation is 0 ; For transfer node $h$, there is one flow sent to $h$, the value of the equation is - 1 . Constraint (3) ensures the flow conservation from transfer node $h$ to destination port $d$. Constraint (4) ensures only one vessel and one transfer node be selected for each cargo od. Constraint (5) ensures $x^{i j}{ }_{\text {od }}=1$ if $x^{h s}{ }_{\text {od }}$ $=1$ and vice versa.

\section{NUMERICAL EXAMPLES}

In this section, a numerical example is provided to demonstrate the applicability of the proposed model and the efficiency of the genetic algorithm for solving the MMNFP. The example is constructed according to the real-world case of intermodal sea-rail network in China. The structure of real intermodal network is shown as follows. 


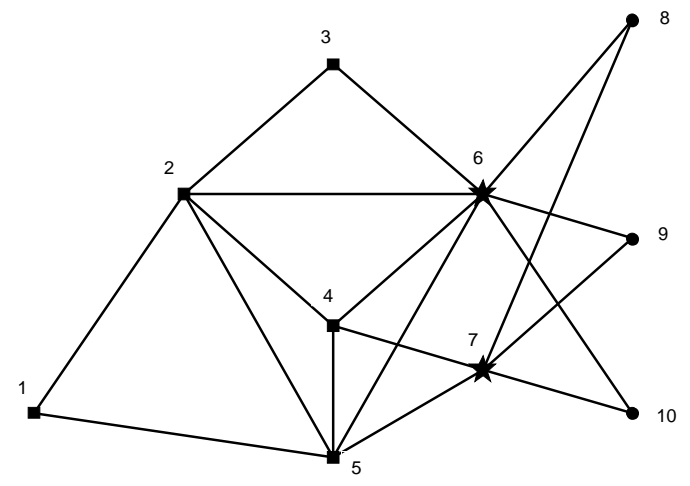

Figure 1. The real intermodal network

As shown in Figure 1, there are 10 nodes and 18 arcs. The black square denotes ordinary nodes; the black star denotes intermodal transfer nodes; the black round denotes the destination ports. The unit transportation cost between any two nodes and the unit transfer cost at intermodal transfers are shown in Table 1. If there is not an arc between two nodes, the transportation cost between the two nodes is infinity.

$\begin{array}{ccccccccccc}\begin{array}{c}\text { Transpor- } \\ \text { ta-tion } \\ \text { cost }\end{array} & 1 & 2 & 3 & 4 & 5 & 6 & 7 & 8 & 9 & 10 \\ 1 & \text { Inf } & 538 & \text { Inf } & \text { Inf } & 412 & \text { Inf } & \text { Inf } & \text { Inf } & \text { Inf } & \text { inf } \\ 2 & 538 & \text { Inf } & 360 & 360 & 632 & 400 & \text { Inf } & \text { Inf } & \text { Inf } & \text { inf } \\ 3 & \text { Inf } & 360 & \text { Inf } & \text { Inf } & \text { Inf } & 360 & \text { Inf } & \text { Inf } & \text { Inf } & \text { inf } \\ 4 & \text { Inf } & 360 & \text { Inf } & \text { Inf } & 300 & 360 & 223 & \text { Inf } & \text { Inf } & \text { Inf } \\ 5 & 412 & 632 & \text { Inf } & 300 & \text { Inf } & 632 & 282 & \text { Inf } & \text { Inf } & \text { Inf } \\ 6 & \text { Inf } & 400 & 360 & 360 & 632 & \text { Inf } & \text { Inf } & 447 & 223 & 538 \\ 7 & \text { Inf } & \text { Inf } & \text { Inf } & 223 & 282 & \text { Inf } & \text { Inf } & 824 & 360 & 223 \\ 8 & \text { Inf } & \text { Inf } & \text { Inf } & \text { Inf } & \text { Inf } & 447 & 824 & \text { Inf } & \text { Inf } & \text { Inf } \\ 9 & \text { Inf } & \text { Inf } & \text { Inf } & \text { Inf } & \text { Inf } & 223 & 360 & \text { Inf } & \text { Inf } & \text { Inf } \\ 10 & \text { Inf } & \text { Inf } & \text { Inf } & \text { Inf } & \text { Inf } & 538 & 223 & \text { Inf } & \text { Inf } & \text { Inf } \\ \text { Transfer } & & & 6 & & & & & 7 & & \\ \text { cost } & & & 500 & & & & & 600 & & \end{array}$

The MMNFP has 21 commodities and their container transportation volume distribution is shown in Table 2.

Unit: TEU

Table 2. Transportation cost and transfer cost

$\begin{array}{cccccccc} & 1 & 2 & 3 & 4 & 5 & 6 & 7 \\ 8 & 55 & 60 & 65 & 63 & 52 & 58 & 64 \\ 9 & 50 & 61 & 52 & 59 & 67 & 54 & 62 \\ 10 & 66 & 63 & 54 & 57 & 59 & 61 & 65\end{array}$

The genetic algorithm is implemented in the numerical example. And the simulation results in iteration 1, $10,20,100$ are shown in Figure 2. As shown in Fig. 2, from the iteration 20, the container routes scenario converges to a stable state. 

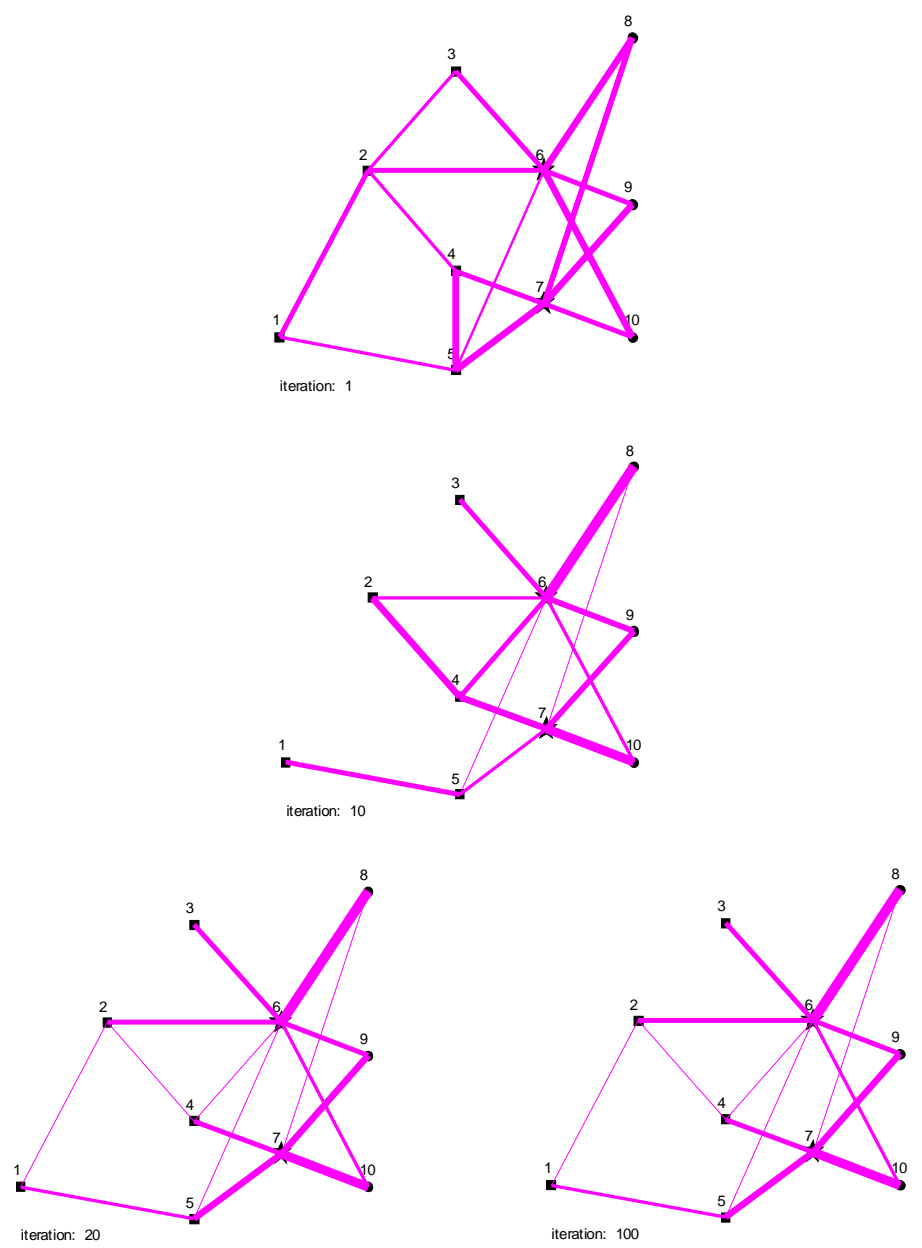

Figure 2. Simulation results

The optimal container routes for each cargo are as follow. As shown in Table 3, the transportation volume on arc $(6,8)$ is the most $(353$ TEUs), which is on the optimal routes of seven cargoes. And the volume on $\operatorname{arc}(7,10)$ is the second most $(310$ TEUs). The arc $(5,8)$ is the least $(52$ TEUs). 
Table 3. The optimal container routes for each cargo

Car- Container route for the cargo

go

od

$1-8 \quad 1 \rightarrow 2 \rightarrow 6 \rightarrow 8$

$1-9 \quad 1 \rightarrow 5 \rightarrow 7 \rightarrow 9$

$1-10 \quad 1 \rightarrow 5 \rightarrow 7 \rightarrow 10$

$2-8 \quad 2 \rightarrow 6 \rightarrow 8$

$2-9 \quad 2 \rightarrow 6 \rightarrow 9$

$2-10 \quad 2 \rightarrow 4 \rightarrow 7 \rightarrow 10$

$3-8 \quad 3 \rightarrow 6 \rightarrow 8$

$3-9 \quad 3 \rightarrow 6 \rightarrow 9$

$3-10 \quad 3 \rightarrow 6 \rightarrow 10$

$4-8 \quad 4 \rightarrow 6 \rightarrow 8$

$4-9 \quad 4 \rightarrow 7 \rightarrow 9$

$4-10 \quad 4 \rightarrow 7 \rightarrow 10$

$5-8 \quad 5 \rightarrow 6 \rightarrow 8$

$5-9 \quad 5 \rightarrow 7 \rightarrow 9$

$5-10 \quad 5 \rightarrow 7 \rightarrow 10$

$6-8 \quad 6 \rightarrow 8$

$6-9 \quad 6 \rightarrow 9$

$6-10 \quad 6 \rightarrow 10$

$7-8 \quad 7 \rightarrow 8$

$7-9 \quad 7 \rightarrow 9$

$7-10 \quad 7 \rightarrow 10$

\section{CONCLUSIONS}

The paper focuses on the multimodal multicommodity network flow problem (MMNFP) to select optimal container routes for each cargo in an intermodal sea-rail network. The problem is complicated by two subproblems: (1) container route selection (2) intermodal transfer selection. Since MMNFP is NP-hard, genetic algorithm is proposed to solve it. To illustrate the proposed model and heuristic algorithm, they are implemented in a small simple intermodal network. Future research is based on the proposed model which will focus on the MMNFP with time windows and random travel time.

\section{REFERENCES}

1)Ziliaskopoulos and W. Wardell, 2003, "An Intermodal optimum path algorithm for multimodal networks with dynamic arc travel times and switching delays, " European Journal of Operational Research, Vol. 125, pp. 486-502.

2)E. H. Miller and H. Mahmassani, 2000, "Least expected time paths in stochastic, time-varying transportation networks, "Transportation Science, Vol. 34, No. 2, pp. 198-215.

3)F. S. Worth and B. E. Peterson, 2000, "Intermodal and international freight network modeling," Transportation Research Part C, Vol. 8, pp. 147-166.

4)H. Hamacher and S. Ruzika, 2006, "Algorithms for time dependent bicriteria shortest path problems," Discrete Optimization, Vol. 3, pp. 238-254.

5)H. Min, 1991, "International intermodal choices via chance-constrained goal programming," Transportation Research Part A, Vol. 25, No. 6, pp. 351-362.

6) J. Berube and J. Potvin, 2006, "Time-dependent shortest paths through fixed sequence of nodes: application to a travel planning problem, "Computers and Operations Research, Vol. 33, pp. 18381856. 
7)L. J. Ji and B. L. Lin, 2011, "Car Flow Assignment and Routing Optimization Model of Railway Network Based on Multi-Commodity Flow Model," China Railway Science, Vol. 32, No. 3, pp. 107-119.

8)P. Pattanamekar and D. Park, 2003, " Dynamic and stochastic shortest path in transportation networks with two components of travel time uncertainty, "Transportation Research C, Vol. 11, No. 5, pp. 331-354.

9)T. S. Chang, 2008, "Best routes selection in international intermodal networks," Computers \& Operations Research, Vol. 35, pp. 2877-2891.

10) Y. Zhang and Y. X. Wang, 2013, "Best routes selection in international intermodal networks," Journal of Dalian Maritime University, Vol. 39, No. 4, pp. 111-114. 